\title{
A Quantitative Performance Analysis of Edge Detectors with Hybrid Edge Detector
}

\author{
Bunil Kumar Balabantaray ${ }^{1 *}$, Om Prakash Sahu ${ }^{1}$, Nibedita Mishra ${ }^{1}$, Bibhuti Bhusan Biswal ${ }^{2}$ \\ 1 Product Design and Development Laboratory, Department of Industrial Design, National Institute of \\ Technology Rourkela-769008, Odisha, India. \\ 2 Department of Industrial Design, National Institute of Technology Rourkela-769008, Odisha, India. \\ * Corresponding author. Tel: +91-9438592903; email: bunil.balabantaray@gmail.com \\ Manuscript submitted November 9, 2015; accepted December 30, 2015. \\ doi: $10.17706 /$ jcp.12.2.165-173
}

\begin{abstract}
Understanding of images via features like edges plays a vital role in many image processing applications. However, obtaining an optimum edge detector that performs well in every possible imaging condition is still an open challenge to researchers. In this paper, a quantitative analysis of some significant state of art edge detection techniques such as Canny's, Prewitt, Sobel, Laplacian of Gaussian, fuzzy based edge detection, wavelet based edge detector with hybrid edge detection technique is proposed based on the correspondence between their outcomes. The hybrid edge detection method utilizes fuzzy logic partitioning along with wavelet transformation to maintain a proper balance in the false detections i.e., false positives and false negatives rates and provides better tracking of edge information. Various subjective as well as objective quality measures are provided for quantitative analysis of edge detectors. The experimental results confirm that compared to other techniques the hybrid edge detection technique outperform in terms of edge detection accuracy exclusively when the images are corrupted by noises.
\end{abstract}

Key words: Edge detection, fuzzy probability, fuzzy partitioning, entropy, wavelet transformation.

\section{Introduction}

Vision system with flexible automation has a significant impact in current trends of manufacturing. Especially while performing assembly operations in the unstructured environment of industry there is a great need of advanced and automated machine vision technology along with the robotic application. In the long run, the robotic platform reduces the operating cost significantly, improves the quality of assembled product and increases the productivity efficiency [1].

Particularly feature detection and feature extraction process is much important to any vision system application such as for object recognition or object tracking. However, edge information is a very critical feature which provides a visual overview of the object that helps in the recognition process. In literature, edge is defined as a boundary present between two dissimilar regions. In other word, edge comes into picture when there is abrupt change in intensity level. In literature variety of edge detector techniques are explained [2] and are normally treated as high pass filters.

Classical edge detection methods follow simple convolution operation of the image within uniform regions. The principal issue of the edge detection is to decide which image pixels is edge and which one is not an edge pixel. This process is normally fuzzy in nature. Consequently several fuzzy based approaches are proposed in literature for edge detection process. However, complexity of uncertainty towards the decision is more in 
the neighborhood of the edge and when the image becomes noisy. In noisy image noise and edge belong to high frequency and it is difficult to distinguish between noise and edge pixel. Normally, efficiency of edge detectors depends upon its detection accuracy in presence of noise or any other degradation.

In this paper, several prominent edge detection techniques are described along with advantages and disadvantages and compared with the hybrid edge detection technique. Section II provides a brief literature review of edge detection techniques. In the Section 3, a brief description about the scope and materials available for the compared edge detection methods in image processing applications are provided. Section 4 provides the performance analysis and different qualitative measures to measure the accuracy of edge detectors. Final section conclude the paper with is future direction.

\section{Background}

In a two dimensional image, pixel whose gray level value is much different from its neighborhood pixels is distinguished as an edge pixel. However, it is difficult to define the word "much" quantitatively and it is quite fuzzy in nature. Besides the quantitative aspect of word much also depends upon the concerned application. Roberts, Sobel, Prewitt, Laplacian of Gaussian and Canny [2] are most popular conventional edge detectors used in various applications. Among a variety of above mentioned edge detector, canny algorithm is much efficient. The Canny edge detector provides a complete edge map with accurate localization and minimal false detection.

Moreover, edge detection using wavelet transform is also an important direction which cannot be ignored. Wavelet transformation provides significant noise resistive capability as well as accurate positioning feature and without minimized effect of false detection [3]. However, bad sensitivity to different edge directions limits its performance. To alleviate the problems related to traditional wavelet based edge detector, Lixia Jiang, Wenjun Zhou and Wang Yu [4] proposed an improved wavelet algorithm. The proposed algorithm reduces the sensitivity to direction properties and results complete edge. Edge detection using mathematical morphology (MM) has a wide range of application in the field of medical imaging.

Various combination of basic morphological operations such as erosion; dilation; opening and closing are employed for the detection of edge. However, the efficiency of the detector solely depends upon the basic structuring element used. A variety of MM based edge detectors are proposed in literature. Moreover, in [5] Yu-Qian, Zhao, Gui Wei-hua, Chen Zhen-cheng, Tang Jing-tian, and Li Ling-yun had proposed a multi scale and multi structured MM edge detector to track a complete edge feature and to overcome the difficulties with conventional edge detection approaches. In [6], S. Lu, Z. Wang and J. Shen had provided an excellent theory based on fuzzy logic to deal with the uncertainty of edge detection problem. W. Barkhoda, F. A. Tab and O. K. Shahryari [7] proposed a new fuzzy based edge detection algorithm. The proposed method reduces the detection error of some unreal edges and compared the result with the standard algorithms. Liming Hu, Cheng, H. D., and Zhang, Ming [8] had uses the concept of edge continuity and proposed a better edge detector. The proposed edge detector was basically controlled by some fuzzy If-Then inference rules. D. K. Patel and S. A. More [9] used fuzzy logic for accurate and noise free edge detection and then it is enhanced using Cellular Learning Automata. B. K. Balabantaray and B. B. Biswal [10] proposed a hybrid edge detector based on fuzzy inference rule along with modified wavelet transform. The Improved wavelet transform can able to track only horizontally and vertically oriented edge pixels. But its quality degrades for noisy images as it is unable to distinguish edge pixels from noise. Fuzzy rule based technique is used as an external filter.

\section{Edge Detection Technique}

Edge detection is a very popular and essential preprocessing step in various image processing applications such as image recognition and image segmentation. However, selection of an optimum and unique edge 
detector is difficult to achieve. Even though the detector follows the same mathematical background they produce different edge maps in different environments. In the following section a variety of edge detector techniques are provided along with their advantages and disadvantages. In literature the conventional edge detectors are grouped into two categories, viz. gradient based and Laplacian based.

\subsection{Sobel Operator}

The Sobel edge detector is a spatial domain gradient based edge detector. The Sobel operator consists of two gradient masks of size $3 \times 3$ one along horizontal direction and another along vertical direction. The pair of mask slides over the image and aims at calculating the gradient at each single pixel of the 2D gray scale image. The detail description about the formulation and edge detection process is defined in [2]. The Sobel operator is a simple edge detector and allows fast computation. The detection process performs a small amount of mathematical calculation and hence makes the detection process computationally cheap. However, the sobel operator has a limited capability to detect the edges in arbitrarily oriented direction. Due to this limited directional capability it only extracts the edge direction only along horizontal and vertical direction. Besides, this edge detector is also not robust to noise. Hence it has limited application where the image is noisy and rich in edges along different directions.

\subsection{Prewitt's Operator}

Like Sobel operator the Prewitt operator also extracts the edges of an image only along vertical and horizontal direction. The detection process follows the same steps as followed by Sobel operator. This operator consists of a pair of masks. The masks are convolved with the image to produce the absolute gradient of the image. The edge strength at a particular pixel is given by the square of the magnitude of the absolute gradients (along $\mathrm{x}$ and $\mathrm{y}$ axis) at the same point. Like Sobel operator the Prewitt edge detector also adopts to only limited directions and hence it has a limited application. The detail description about this edge detector operator is found in [1].

\subsection{Robert's Cross Operator}

It is one of the oldest and most popular edge detection operator. The operator calculates the spatial gradient at each and every pixel of the image under consideration. Absolute magnitude of the gradient at each single pixel of the input image is the resultant edge image. Unlike Prewitt and Sobel operator this operator is quite simple and much faster. Due to its fast computation and easy inclusion, it has a frequent application in hardware implementation.

\subsection{Laplacian-Based Edge Detection}

Laplacian based edge detector is based on the second order derivative approach and it typically behaves as high pass filter. The second order derivative of the gray scale image intensity is calculated and the edge point is localized based on the zero crossing of the derivative. However, the second order derivative is prone to noise and hence prior to edge detection noise removal must be done. A Gaussian low pass filter is convolved with the noisy input image to remove the noise. The convolution process not only filters the isolated noise points and unwanted structures but also blurs the edge structure of the input image. After noise removal the edge point is selected based on the maximum second order gradient value. A predefined threshold value is used to avoid the detection of false edge points. The zero crossings of the pixels whose first order gradient is more than the threshold value is selected as valid edge point and others pixels fall as non-edge pixels. Due to the high pass filtering behavior, this operator is not applicable to noisy environment. As it get confused which high frequency component is edge information and which one is the unwanted noise.

\subsection{Canny's Edge Detection Algorithm}


Among a variety of above mentioned conventional edge detectors canny edge detector is the optimum one. It is robust to noise and it performs multilevel thresholding based on the image characteristics to avoid loss of information and to reduce the false detection rate. The detail description is found in [1]. Unlike above mentioned edged detectors this detector has better edge tracking capability in various directions. However, and Canny edge detector is computationally heavy as compared to other edge detectors. Besides, efficiency of this edge detector is controlled by the selection of thresholds. Selection of threshold value has a mere impact on the accuracy of edge detection and edge point localization. With the advance of one, the accuracy of other one deteriorates. Hence a proper strategy for the selection of threshold values is a major concern in this edge detection process. These factors make this edge detector complex. Due to the above mentioned disadvantages the detector gives poor results for images with complicated imaging conditions.

\subsection{Mathematical Morphology Based Edge Detection}

Mathematical morphology based edge detection approaches outperform for geometrical structure analysis of image. It is based on the principle of set theory. It has primarily applied for binary images but now it has extended its application for gray scale images also. The geometrical structure of the image is analyzed by the help of small pattern i.e., structuring element. Various basic MM operations are performed to reduce the effect of noises and detect the edges. However, the detection process is a function of shape and size of the structuring element. This process adapts non-linear image operators to detect the geometrical and topological structures of the underlying image. It employs direct image manipulation and hence it can be directly used in hardware implementation. Besides, as compared to traditional edge detectors, the MM based edge detectors provide efficient noise suppression and accurate edge tracking. However, this type of edge detectors has limited capability to track the edge information for images rich in geometrical shape.

\subsection{Wavelet Based Edge Detection}

Wavelet based edge detection methods are popular in literature due to its multi scale edge tracking capability. However, these types of detectors have limited capability to directional properties in the detection process. Hence for the improvement of performance, a variety of approaches are proposed in literature to minimize the directional the insensitivity of the conventional approaches.

In [10], a detail description about the disadvantages with the traditional wavelet based edge detection is described and a hybrid approach was proposed by using the concept of fuzzy along with the wavelet transform. The hybrid approach has a better capability to track the more edge information along arbitrarily oriented edge directions.

\subsection{Hybrid Edge Detector}

This edge detection technique uses fuzzy rule along with wavelet theory to detect edge. The hybridization process implements the multi scale edge tracking behavior of wavelet transform and removes its inherent limitation i.e., insensitive to edge direction by using the fuzzy theory. Along with edge preservation the fuzzy theory also helps to filtered out the noises and reduces the false detection rate. Consequently the hybrid edge detector has a capability to detect edges in arbitrarily directions, preserves more image details and is robust to noise.

In basic steps employed in the hybrid edge detection process is described in Fig. 1. The directional of edge points are decided by the fuzzification process. Fuzzy if-then rules are applied to the gray scale image to find out the directional edge points such as edge points along horizontal, vertical and diagonal directions. The fuzzification process is followed by wavelet transform. The fuzzified image from the fuzzification process passes through the wavelet transformation to provide the edges. The next step is to remove the noise and false edge points detected in the previous steps. A mask of size $3 \times 3$ is passed over the output image from the 
previous step. The sliding process continues until the whole image is not scanned. In the scanning process the falsely detected edge pixels are removed and after successful completion of the scanning process we will get the final edge of the image. The detail description of the hybrid edge detector is provided in [7].

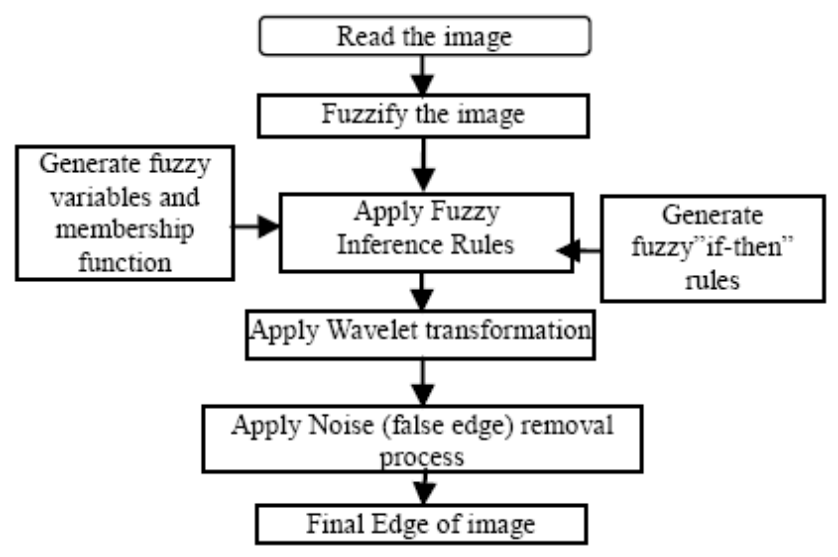

Fig. 1. Hybrid edge detection technique.

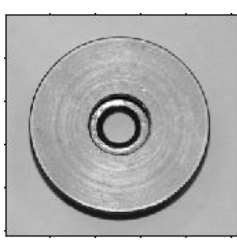

(a)

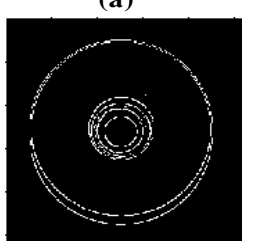

(d)

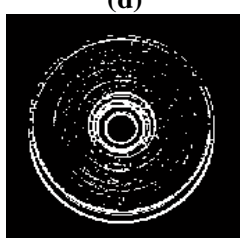

(g)

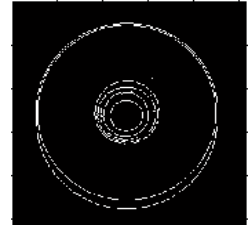

(b)

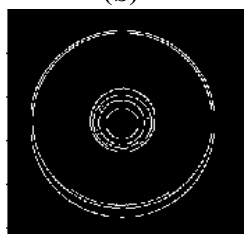

(e)

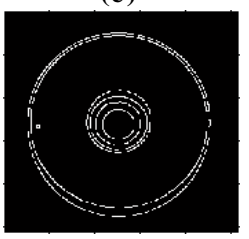

(h)

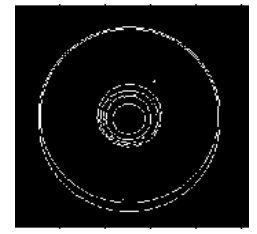

(c)

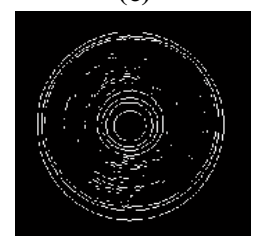

(f)

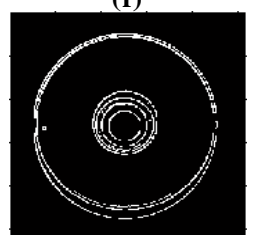

(i)

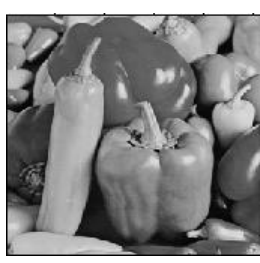

(a)
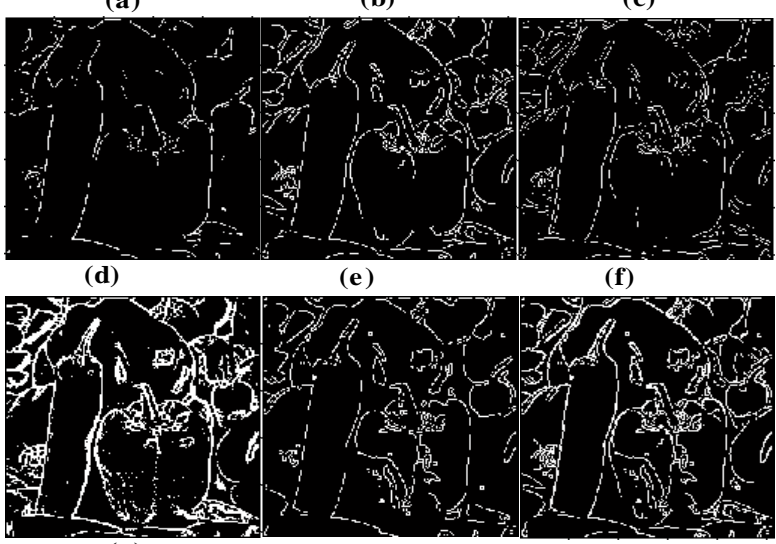

(g)

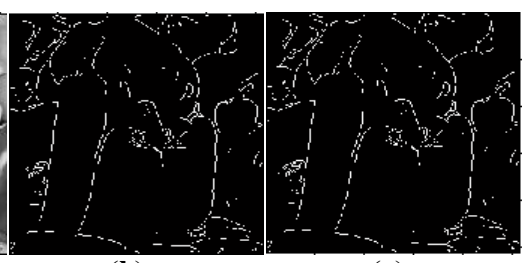

(c)

(i)

Fig. 2. Comparison of results for image "Part.jpg" (a) Original image, (b) Prewitt, (c) Sobel, (d) Roberts, (e)

Fig. 3. Comparison of results for image "Peppers.tiff" (a) Original image, (b) Prewitt, (c) Sobel, (d) Roberts, (e) Canny, (f) Log, (g) MM, (h) Wavelet (i) Hybrid.

\section{Performance Analysis Measure}

This section provides the performance analysis of different edge detectors. The quantitative performance evaluation of different edge detectors are tested with three ground truth test images such as: Part.jpg, Peppers.tiff, Baby.jpg. The whole simulation work is performed in MATLAB (R2015a). The performance analysis of eight edge detector i.e., Prewitt, Sobel, Roberts, Canny, LoG, Mathematical morphology (MM), Wavelet and Wavelet + fuzzy (Hybrid) are presented here to provide a comparison analysis.

Figs. 2-4 are provided to show the visual results obtained from different edge detectors. From the visual perception it is quite clear that for each and every test image the hybrid edge detector provides a better edge detection. 


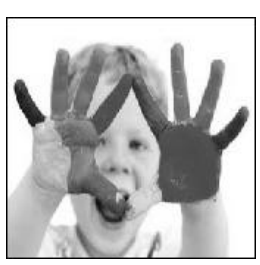

(a)

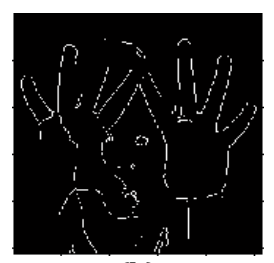

(b)

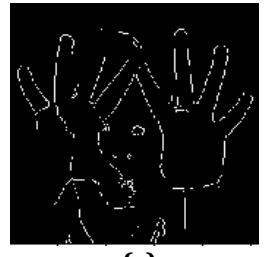

(c)

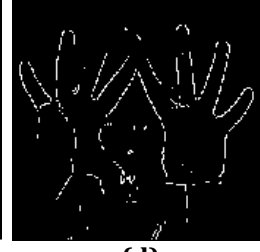

(d)

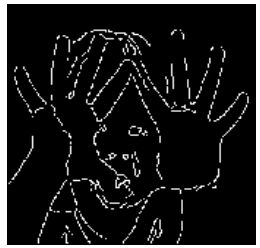

(e)

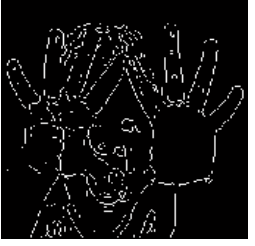

(f)

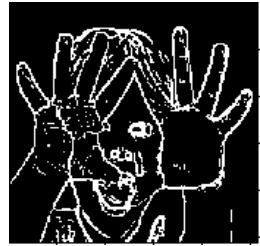

(g)

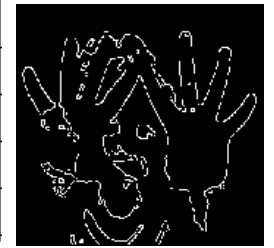

(h)

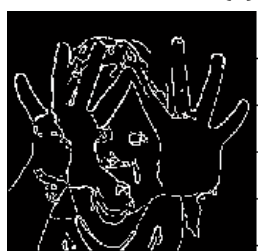

(i)

Fig. 4. Comparison of results for image "Baby.jpg" (a) Original image, (b) Prewitt, (c) Sobel, (d) Roberts, (e) Canny, (f) Log, (g) MM, (h) Wavelet (i) Hybrid.

Besides visual analysis two significant metrics, sensitivity and specificity are also presented here to characterize the accuracy of the edge detectors. These two metrics allows to recognize true edges and not to recognize the false alarms. Sensitivity of an edge detector characterizes the probability of detection of a true edge as an edge pixel. Whereas, probability of detection of a false edge as a non-edge pixel is characterized by the term sensitivity. The sensitivity of an edge detector is normally expressed in terms of true-positive rate $\left(T P_{\text {Rate }}\right)$ and specificity in term of false-positive rate $\left(F P_{\text {Rate }}\right)$. Eq. 1 . Provides the mathematical formulation for true positive rate and Eq. 2 represents the calculation of the false-positive rate.

$$
\begin{gathered}
\text { Sensitivity }=T P_{\text {Rate }}=\frac{T_{\text {positive }}}{\left(T_{\text {positive }}+F_{\text {positive }}\right)} \\
\text { Specificity }=F P_{\text {Rate }}=1-\left(\frac{T_{\text {negative }}}{T_{\text {negative }}+F_{\text {positive }}}\right)
\end{gathered}
$$

where, $T_{\text {positive }}$ denotes the true positive i.e. the number of edge pixels correctly detected. Eq. 3 provides the probability of true-positive $\left(P T_{\text {positive }}\right)$ outcome of an image of size say $(M \times N)$ mathematically.

$$
p_{T_{\text {positive }}}=\operatorname{mean}\left(X_{(m, n)} . Y_{(m, n)}\right)
$$

$X(m, n)$ denotes the probability of a pixel to be a true edge where $m=1, \ldots, M$ and $n=1, \ldots, N . Y(m, n)$ represent the probability of a pixel to be detected as edge. In the similar way the number of pixels which are erroneously classified as edge pixels are referred as false positive $\left(F_{\text {positive }}\right)$ and the edge pixels that are not classified as edge pixel, called as false negative ( $\left.F_{\text {negative }}\right)$. In order to calculate all these parameters for the comparison of different edge detectors an ideal edge map is to be necessarily defined. However defining an ideal map is unclear therefore in the present paper the edge map obtained from the hybrid edge detector is considered as an ideal edge map for the calculation of different parameters. Tables 1-2 represents the comparison of percentage of correct detection $\left(P_{\text {cod }}\right)$ and percentage of pixels that were not detected $\left(P_{\text {nod }}\right)$ respectively. Percentage of false alarm $P_{\text {fal }}$ (i.e. percentage of pixels that are erroneously detected as edge pixels) for different edge detectors is plotted in Fig. 5.

$$
p_{\text {cod }}=\frac{T_{\text {positive }}}{\operatorname{Max}\left(E_{I}, E_{D}\right)} \times 100
$$




$$
\begin{gathered}
p_{\text {nod }}=\frac{F_{\text {negative }}}{\operatorname{Max}\left(E_{I}, E_{D}\right)} \times 100 \\
p_{\text {fal }}=\frac{F_{\text {positive }}}{\operatorname{Max}\left(E_{I}, E_{D}\right)} \times 100
\end{gathered}
$$

where the ideal edge points of the image is presented by $E_{I}$ and the number of edge points detected is defined by $E_{D}$.

Table 1. Comparison of $P_{\text {cod }}$ Using Different Edge Detectors

\begin{tabular}{lccc}
\hline \hline Images & Part & Peppers & Baby \\
\hline Prewitt & .5401 & .3019 & .3362 \\
Roberts & .5424 & .3033 & .3372 \\
Roberts & .6226 & .3046 & .3667 \\
Canny & .7025 & .6099 & .6152 \\
LoG & .5631 & .4600 & .5936 \\
MM & .5341 & .5432 & .6139 \\
Wavelet & .6840 & .5798 & .6048 \\
Hybrid & .7432 & .8103 & .7799 \\
\hline \hline
\end{tabular}

Table 2. Comparison of $P_{\text {nod }}$ Using Different Edge Detectors

\begin{tabular}{llll}
\hline \hline Images & Part & Peppers & Baby \\
\hline Prewitt & .4439 & .7506 & .6932 \\
Roberts & .4419 & .7482 & .6917 \\
Roberts & .3803 & .7460 & .6474 \\
Canny & .3343 & .3517 & .3500 \\
LoG & .3802 & .5136 & .3717 \\
MM & .4471 & .3981 & .4335 \\
Wavelet & .3418 & .3825 & .3601 \\
Hybrid & .3236 & .1897 & .2001 \\
\hline \hline
\end{tabular}

Table 3. Comparison of Detection Error Using Different Edge Detectors

\begin{tabular}{llll}
\hline \hline Images & Part & Peppers & Baby \\
\hline Prewitt & .5401 & .3019 & .3362 \\
Roberts & .5424 & .3033 & .3372 \\
Roberts & .6226 & .3046 & .3667 \\
Canny & .7025 & .6099 & .6152 \\
LoG & .5631 & .4600 & .5936 \\
MM & .5341 & .5432 & .6139 \\
Wavelet & .6840 & .5798 & .6048 \\
Hybrid & .7432 & .8103 & .7799 \\
\hline \hline
\end{tabular}

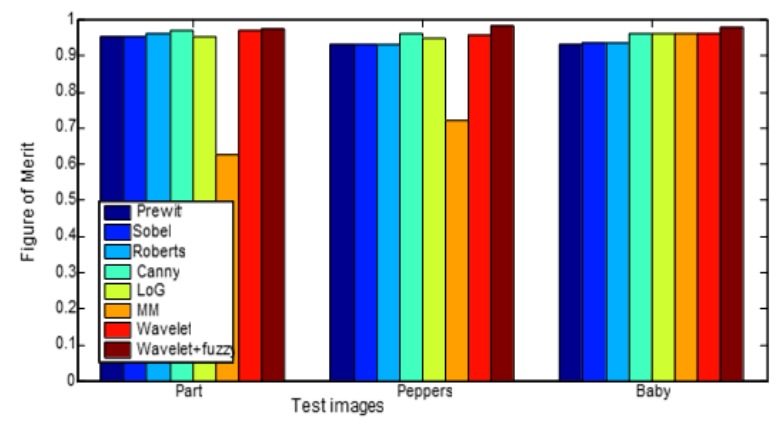

Fig. 5. Percentage of false alarm for different edge detectors.

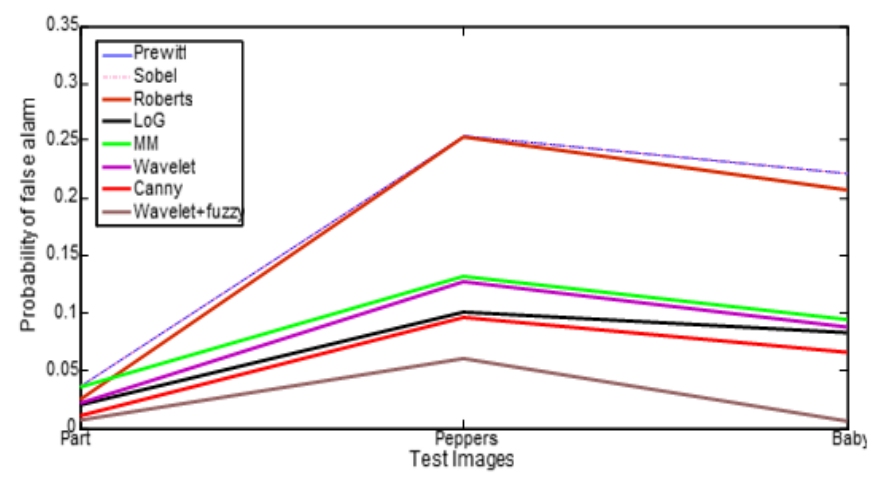

Fig. 6. FoM for different edge detectors.

Table 3 presents the comparison of detection error of different edge detectors. The detection error of an edge detector is expressed by the Eq. 7 . The difference between the of the false-positive rate $\left(F P_{\text {Rate }}\right)$ and true positive rate $\left(T P_{\text {Rate }}\right)$ of concerned edge detector is termed as the detection error.

Pratt had proposed figure of merit (FoM), an another important measure for accessing the performance measure of edge detectors. The gap between all pairs of points corresponding to the ground truth and the estimated edge map is used for the calculation of FoM. Plot of FoM for different edge detectors are presented in Fig. 6. The mathematical formula for the calculation of FoM is given in Eq. 8.

$$
\begin{aligned}
& \text { Detection error }=\sqrt{\left(1-T P_{\text {Rate }}\right)^{2}+\left(F P_{\text {Rate }}\right)^{2}} \\
& F O M=\frac{1}{\operatorname{Max}\left(E_{G}, E_{E}\right)} \sum_{i=1}^{E_{E}} 1 / 1+\alpha \cdot d_{i}^{2}
\end{aligned}
$$


where $E_{G}$ and $E_{E}$ represents the number of edge points on the ideal ground truth and the calculated edge map respectively. $d i$ measures the distance between $i^{\text {th }}$ edge pixel of the estimated and the ground truth edge map. $\alpha$ is the constant coefficient and considered as $1 / 9$ in this paper. The range of the FoM varies in the range of $[0,1]$. The optimal value of the FoM is 1 which represents that the detected edges coincides with the ground truth.

\section{Conclusion}

In this paper a quantitative analysis to evaluate the performance of different state of art edge detectors in literature is proposed. Different objective and subjective quality measures are provided here to analyze the performance of edge detectors. In the experiment, several significant edge detectors are compared with the hybrid edge detector. Along with the visual perception of the compared edge detectors several quantitative analysis measures such as sensitivity, specificity, $P_{\text {cod }}, P_{\text {nod }} P T_{\text {positive, }}$ detection error and FoM are employed here for the analysis. From the visual analysis, the hybrid edge detector outperforms the other compared edge detectors in terms of better edge localization and edge detection. Moreover, it is also found that the quantitative analysis measures of the hybrid edge detector are better than other compared methods. The quantitative measures completely agree with the visual results hence can be used further. Finally an edge detector characterized by lower detection error and higher FoM is considered to be a best detector for detecting edges.

\section{References}

[1] Cid, J. M., \& Reyes, F. C. (2009). Visual servoing controller for robot manipulators. Proceedings of International Conference on Electrical, Communications, and Computers (pp. 153-158).

[2] Balabantaray, B. K., Das, B., \& Biswal, B. B. (2014). Comparison of edge detection algorithm for part identification in a vision guided robotic assembly system. Book Chapter in Soft Computing Techniques in Engineering Applications, Studies in Computational Intelligence, 543, 183-206.

[3] Li, J. (2003). A Wavelet Approach to Edge Detection. PhD diss., Sam Houston State University.

[4] Lixia, J., Wenjun, Z., \& Yu, W. (2010). Study on improved algorithm for image edge detection. Proceedings of The 2nd International Conference on Computer and Automation Engineering (pp. 476-479).

[5] Zhao, Y.-Q., Gui, W.-H., et al. (2006). Medical images edge detection based on mathematical morphology. Proceedings of IEEE-EMBS 27th Annual International Conference of the Engineering in Medicine and Biology Society (pp. 6492-6495).

[6] Wang, Z., \& Shen, J. (2003). Neuro-fuzzy synergism to the intelligent system for edge detection and enhancement. Pattern Recognition, 36(10), 2395-2409.

[7] Barkhoda, W., \& Shahryari, O.-K., (2003). (2009). Fuzzy edge detection based on pixel's gradient and standard deviation values. Proceedings of International Multiconference on Computer Science and Information Technology (pp. 12-14).

[8] Hu, L.-M., Cheng, H. D., \& Zhang, M. (2007). A high performance edge detector based on fuzzy inference rules. Information Science, 177(21), 4768-4784.

[9] Patel, D. K., \& More, S. A. (2013). Edge detection technique by fuzzy logic and Cellular Learning automata using fuzzy image processing. Proceedings of International Conference on Computer Communication and Informatics (pp. 1-6).

[10] Balabantaray, B. K., \& Biswal, B. B. (2014). Hybrid edge detection technique for part identification in robotic assembly system under vision guidance. Proceedings of 5th International and 26th All India Manufacturing Technology, Design and Research Conference (pp. 1-6). 


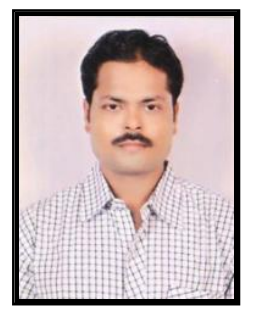

Bunil Kumar Balabantaray was born in Cuttack, Odisha, India in 1984. He received the B.E. degree in information technology in 2005 and M.Tech. in computer science and engineering in 2010 from Biju Patnaik University of Technology (BPUT), Rourkela, Odisha, India. Currently he is perusing PhD at National Institute of Technology Rourkela, India.

He has worked as Lecturer in B.C.E.T. (under BPUT), Odisha, India. Then he worked as Assistant Professor at DRIEMS (under BPUT), Odisha, India from 2005 to 2010. Then he worked as Assistant Professor at Synergy Institute of Technology (under BPUT), Odisha, India from 2010 to 2012. He has published more than 13 technical research papers at national and international levels journal and conferences and two book chapters in edited book in Springer. His areas of research include robotics, machine vision system, and image processing.

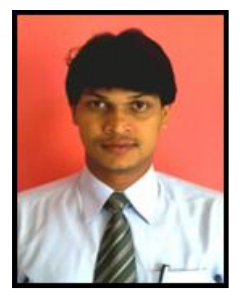

Om Prakash Sahuhor was born in Bhilai, India in 1982. He received the B.E. degree in electronics and telecommunication in 2005 and M-Tech. in Instrumentation and control system in 2008 from the University of CSVT, Bhilai, India. Currently he is pursuing a PhD at National institute of technology Rourkela, India.

He joined as a faculty in RCEE from 2006 to 2012. He has published more than 18 technical research papers at national and international levels. His areas of research interest include industrial robotics, control system and instrumentation, computer integrated manufacturing and automation.

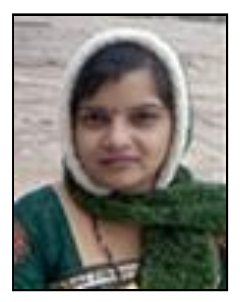

Nibedita Mishra was born in Talcher, Odisha, India, in 1985. She received the B.E. degree in mechanical engineering in 2006 and M.Tech. in production engineering in 2011 from the BPUT, Odisha, India. Currently she is perusing a PhD at National Institute of Technology Rourkela, India.

She has working experience as a faculty from Nov. 2006 to Oct. 2012. She has published more than 3 technical research papers at National and International levels. Her areas of research include human robot interaction, assistive robots and service robots.

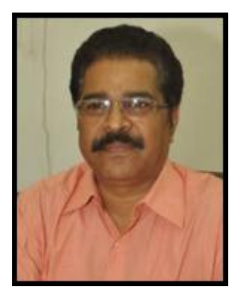

Bibhuti Bhusan Biswal was born in Rourkela, India in 1962. He received the B.E. degree in mechanical engineering from UCE, Burla, India in 1985. Subsequently he completed his M.Tech, and Ph.D. from Jadavpur University, Kolkata.

He joined as a faculty of Mechanical Engineering at UCE Burla from 1986 and continued till 2004 and then joined National Institute of Technology, Rourkela as Professor and currently he is a Professor in the Department of Industrial Design and also the dean (Faculty Welfare) of NIT Rourkela. He has been actively involved in various research projects and published more than 110 papers at National and International levels including a monogram on Management Information System for ISTE, New Delhi, and 6 book chapters and having more than 3 patents. His areas of research interest include robotics, FMS, CAD/CAM, mechatronics, CIM, AMT, virtual manufacturing, and production management. 